\title{
TYPE-2 FUZZY G-TOLERANCE RELATION AND ITS PROPERTIES
}

\author{
MAUSUMI SEN ${ }^{1, *}$, DHIMAN DUTTA $^{1}$ AND ASHOK DESHPANDE ${ }^{2}$
}

\begin{abstract}
In this short communication we generalize the definition of type-2 fuzzy tolerance relation and consequently the type-2 fuzzy G-tolerance relation in type- 2 fuzzy sets. The type- 2 fuzzy Gtolerance relation helps in finding the type-2 fuzzy G-equivalence relation. Moreover, we have studied the notion of type- 2 fuzzy tolerance relation in abstract algebra.
\end{abstract}

\section{INTRODUCTION}

Type-2 fuzzy sets are relatively new in the world of fuzzy sets and systems. Although they were originally introduced in 1975 by L. A. Zadeh [21], type-2 sets did not gain popularity until their reintroduction by J. M. Mendel [12]. These newer fuzzy sets were now thought of as an extension of the already popular fuzzy sets (now labelled type-1) to include additional uncertainties in the set. Type-2 fuzzy sets are unique and conceptually appealing, because they are fuzzy extension rather than crisp. Type-2 fuzzy sets have membership functions as type- 1 fuzzy sets. The advantage of type- 2 fuzzy sets is that they are helpful in some cases where it is difficult to find the exact membership functions for a fuzzy sets. There are wide variety of applications of type-2 fuzzy sets in science and technology like computing with words [14], human resource management [9], forecasting of time-series [11], clustering [1,17], pattern recognition [3], fuzzy logic controller [20], industrial application [4], simulation [18], neural network [2], [19], and transportation problem [13].

The concept of cartesian product of type-2 fuzzy sets was given by Hu et al. [10] as an extension of type-1 fuzzy sets. The properties of membership grades of type-2 fuzzy sets and set-theoretic operations of such sets have been studied by Mizumoto and Tanaka [15], [16]. Dubois and Prade [6][8] discussed the composition of type-2 fuzzy relations and presented a formulation only for minimum t-norm which is, perhaps, an extension of type-1 sup-star composition. Type-2 fuzzy relations (T2 FRs in short) were introduced in [12]. There are different kinds of applications of fuzzy relations in the theory of type-1 fuzzy sets. The other motivation of this research is to investigate T2 FRs and their compositions. A type-2 fuzzy tolerance relation is a type-2 fuzzy reflexive and symmetric relation, but not necessarily transitive relation.

The main objective of this paper is to generalize the definition of type-2 fuzzy tolerance relation and consequently the type-2 fuzzy G-tolerance relation in type-2 fuzzy sets. The type-2 fuzzy G-tolerance relation helps in finding the type-2 fuzzy G-equivalence relation. We have studied the concept of type-2 fuzzy tolerance relation in abstract algebra.

The paper is organized as follows: Section (2) introduces some basic definitions related to the concept. We have discussed type-2 fuzzy G-tolerance relation and type-2 fuzzy G-equivalence relation in Section (3). Section (4) deals with the conversion of type-2 fuzzy tolerance relation. Section (5) describes type-2 fuzzy tolerance relation in algebraic structures.

\section{Preliminaries}

Let us define some preliminary concepts in this section.

Received $26^{\text {th }}$ July, 2017; accepted $9^{\text {th }}$ October, 2017; published $1^{\text {st }}$ November, 2017.

2010 Mathematics Subject Classification. 03E72.

Key words and phrases. type-2 fuzzy G-tolerance relation; type-2 fuzzy G-equivalence relation; type-2 fuzzy G-preclass; type-2 fuzzy G-tolerance class.

(C)2017 Authors retain the copyrights of their papers, and all open access articles are distributed under the terms of the Creative Commons Attribution License. 
Definition 1. [21] Let $X$ be a non-empty universe. Then a mapping $A: X \rightarrow F([0,1])$ is called a type-2 fuzzy set (T2 FS, for short) on $X$, characterized by a membership function $\mu_{\tilde{A}}(x): X \rightarrow F([0,1])$ and is expressed by the following set notation : $\tilde{A}=\left\{\left(x, \tilde{\mu}_{\tilde{A}}(x)\right): x \in X\right\} . F([0,1])$ denotes the set of all type-1 fuzzy sets that can be defined on the set $[0,1] . \mu_{\tilde{A}}(x)$, itself is a type-1 fuzzy set for value of $x \in X$ and is characterized by a secondary membership function $f_{x}: J \rightarrow[0,1]$. Therefore, $\tilde{A}$ can be represented as : $\tilde{A}=\left\{\left\langle x,\left\{\left(u, f_{x}(u)\right): u \in J\right\}\right\rangle: x \in X\right\}$, where $J \subseteq[0,1]$ is the set of all possible primary membership functions corresponding to an element $x$.

Let $\mu_{A}(x)$ and $\mu_{B}(x)$ be the secondary membership functions of two type-2 fuzzy sets in the universal set $X$. The operations of union, intersection and complement of two type-2 fuzzy sets $A$ and $B$ using the Zadeh's Extension Principle [21] are defined below:

$$
\begin{aligned}
& \text { Union: } \quad A \cup B=\mu_{A}(x) \sqcup \mu_{B}(x) \\
& =\sum_{u \in J} \frac{f_{x}(u)}{u} \sqcup \sum_{w \in J} \frac{f_{x}(w)}{w} \\
& =\sum_{u, w \in J} \frac{f(u) \wedge g(w)}{u \vee w} \\
& \text { Intersection: } \quad A \cap B=\mu_{A}(x) \sqcap \mu_{B}(x) \\
& =\sum_{u \in J} \frac{f_{x}(u)}{u} \sqcap \sum_{w \in J} \frac{f_{x}(w)}{w} \\
& =\sum_{u, w \in J} \frac{f(u) \wedge g(w)}{u \wedge w} \\
& \text { Complement: } \quad \bar{A}=\neg \mu_{A}(x) \\
& =\sum_{u \in J} \frac{f_{x}(u)}{1-u} \\
& \text { Containment: } A \sqsubseteq B(A \sqsupseteq B) \text { If } \mu_{A}(x) \leq \mu_{B}(x)\left(\mu_{A}(x) \geq \mu_{B}(x)\right)
\end{aligned}
$$

where $\sqcup$ is denoted as join, $\sqcap$ is denoted as meet, $\neg$ is denoted as negation operator, $\vee$ is denoted as max and $\wedge$ is denoted as min operator as defined by Mizumoto and Tanaka [16] in type-2 fuzzy sets respectively.

Definition 2. [12] The cartesian product of two type-2 fuzzy sets, say $A$ and $B$ on $X$ is defined as $(A \times B)(x, y)=A(x) \wedge B(y)$ for all $x, y \in X$.

Definition 3. [12] Let $A, B$ be two type-2 fuzzy sets on $X$ and $Y$ respectively, then the type-2 fuzzy relation (T2 FR in short) of type-2 fuzzy sets $A \times B$ is the type-2 fuzzy subset of $X \times Y$.

\section{Type-2 Fuzzy G-equivalence Relation}

Type-2 fuzzy reflexive, symmetric and transitive relations were discussed in [10].

Definition 4. Let $\tilde{Q}$ be a T2 FR on $X$. Then $\tilde{Q}$ is said to be type-2 fuzzy

(1) reflexive if $\tilde{Q}(x, x)=\overline{1}$ for all $x \in X$.

(2) antireflexive if $\tilde{Q}(x, x)=\overline{0}$ for all $x \in X$.

(3) weakly reflexive if $\tilde{Q}(x, y) \sqsubseteq \tilde{Q}(x, x)$ and $\tilde{Q}(y, x) \sqsubseteq \tilde{Q}(x, x)$ for all $x, y \in X$.

(4) symmetric if $\tilde{Q}(x, y)=\tilde{Q}(y, x)$.

(5) antisymmetric if $\tilde{Q}$ satisfies $\tilde{Q}(x, x)=\overline{0}$ or $\tilde{Q}(x, x)=\overline{0}$ for all $x, y \in X(x \neq y)$.

(6) transitive if $\tilde{Q} \circ \tilde{Q} \sqsubseteq \tilde{Q}$, where $\tilde{Q} \circ \tilde{Q}$ is defined by

$\tilde{Q} \circ \tilde{Q}(x, y)=\operatorname{sunp}_{z \in X}\{\tilde{Q}(x, z) \wedge \tilde{Q}(z, y)\}$.

Dhiman et al. [5] have extended the definitions of type-2 fuzzy reflexive relation and type-2 fuzzy equivalence relations. 
Definition 5. $[5] \tilde{Q}$ is said to be type-2 fuzzy G-reflexive if

(i) $\overline{0}<\tilde{Q}(x, x)<\overline{1}$, where $\tilde{Q}(x, x)$ lies between $(\overline{0}, \overline{1})$;

(ii) $\tilde{Q}(x, y)<\inf _{t \in X} \tilde{Q}(t, t)$ for all $x \neq y$ in $X$.

$\overline{0}$ and $\overline{1}$ memberships are denoted as $1 / 0$ and $1 / 1$ in type- 2 fuzzy sets respectively. $\overline{0}$ membership in a type-2 fuzzy set means that it has a secondary membership equal to 1 corresponding to the primary membership of 0 , and if it has all other secondary memberships equal to 0 . Similarly, the meaning of $\overline{1}$ is same as $\overline{0}$.

Definition 6. A type-2 fuzzy relation $\tilde{Q}$ in $X$ is a type-2 fuzzy $G$-tolerance relation in $X$ if $\tilde{Q}$ is type-2 fuzzy $G$-reflexive and symmetric in $X$.

Definition 7. [5] A type-2 fuzzy relation $\tilde{Q}$ in $X$ is a type-2 fuzzy G-equivalence relation in $X$ if $\tilde{Q}$ is type-2 fuzzy G-reflexive, symmetric and transitive in $X$.

Example 1. Suppose that in a biotechnology experiment, three potentially new strains of bacteria have been detected in the area around an anaerobic corrosion pit on a new aluminum-lithium alloy used in the fuel tank of a new experiment aircraft. In a pair wise comparison, the following similarity relation $\tilde{Q}$ is developed. For example, the first strain (column 1) has a strength of similarity to the second strain of $\frac{0.1}{0.1}+\frac{0.2}{0.2}$, to the third strain a strength of $\frac{0.4}{0.4}+\frac{0.5}{0.5}$. Hence,

$$
\tilde{Q}=\left(\begin{array}{ccc}
\frac{1}{1}+\frac{0.9}{0.9} & \frac{0.1}{0.1}+\frac{0.2}{0.2} & \frac{0.4}{0.4}+\frac{0.5}{0.5} \\
\frac{0.1}{0.1}+\frac{0.2}{0.2} & \frac{1}{1}+\frac{0.9}{0.9} & \frac{0.6}{0.7}+\frac{0.6}{0.6} \\
\frac{0.4}{0.4}+\frac{0.5}{0.5} & \frac{0.6}{0.7}+\frac{0.6}{0.6} & \frac{0.7}{0.7}+\frac{0.8}{0.8}
\end{array}\right) .
$$

Clearly, (i) $\tilde{Q}(x, x)>\overline{0}$

(ii) $\tilde{Q}(x, y) \sqsubseteq \inf _{t \in X} \tilde{Q}(t, t)$ for all $x \neq y$ in $X$.

Therefore, $\tilde{Q}$ is a type-2 fuzzy $G$-reflexive relation.

Again, $\tilde{Q}(x, y)=\tilde{Q}(y, x)$ for all $x, y \in X$.

Hence, $\tilde{Q}$ is a type-2 fuzzy symmetric relation.

Now,

$$
\tilde{Q} \circ \tilde{Q}=\left(\begin{array}{ccc}
\frac{1}{1}+\frac{0.9}{0.9} & \frac{0.1}{0.1}+\frac{0.2}{0.2} & \frac{0.4}{0.4}+\frac{0.5}{0.5} \\
\frac{0.1}{0.1}+\frac{0.2}{0.2} & \frac{1}{1}+\frac{0.9}{0.9} & \frac{0.6}{0.7}+\frac{0.6}{0.6} \\
\frac{0.4}{0.4}+\frac{0.5}{0.5} & \frac{0.6}{0.7}+\frac{0.6}{0.6} & \frac{0.7}{0.7}+\frac{0.8}{0.8}
\end{array}\right) \circ\left(\begin{array}{ccc}
\frac{1}{1}+\frac{0.9}{0.9} & \frac{0.1}{0.1}+\frac{0.2}{0.2} & \frac{0.4}{0.4}+\frac{0.5}{0.5} \\
\frac{0.1}{0.1}+\frac{0.2}{0.2} & \frac{1}{1}+\frac{0.9}{0.9} & \frac{0.6}{0.7}+\frac{0.6}{0.6} \\
\frac{0.4}{0.4}+\frac{0.5}{0.5} & \frac{0.6}{0.7}+\frac{0.6}{0.6} & \frac{0.7}{0.7}+\frac{0.8}{0.8}
\end{array}\right)
$$

Considering the composition of first row of first matrix and second column of second matrix, we get the first element of the second column of the new matrix which is calculated as follows:

$\left(\frac{1}{1}+\frac{0.9}{0.9}\right) \prod\left(\frac{0.1}{0.1}+\frac{0.2}{0.2}\right)=\frac{0.1}{0.1}+\frac{0.2}{0.2}+\frac{0.1}{0.1}+\frac{0.2}{0.2}=\frac{0.1}{0.1}+\frac{0.2}{0.2}$

Similarly, $\left(\frac{0.1}{0.1}+\frac{0.2}{0.2}\right) \prod\left(\frac{1}{1}+\frac{0.9}{0.9}\right)=\frac{0.1}{0.1}+\frac{0.2}{0.2}$

Again, $\left(\frac{0.4}{0.4}+\frac{0.5}{0.5}\right) \prod\left(\frac{0.6}{0.7}+\frac{0.6}{0.6}\right)=\frac{0.4}{0.4}+\frac{0.4}{0.4}+\frac{0.5}{0.5}+\frac{0.5}{0.5}=\frac{0.4}{0.4}+\frac{0.5}{0.5}$

Now, $\left[\left(\frac{0.1}{0.1}+\frac{0.2}{0.2}\right) \sqcup\left(\frac{0.1}{0.1}+\frac{0.2}{0.2}\right)\right] \sqcup\left(\frac{0.4}{0.4}+\frac{0.5}{0.5}\right)$

$=\left(\frac{0.2}{0.4}+\frac{0.2}{0.5}\right)>\left(\frac{0.1}{0.1}+\frac{0.2}{0.2}\right)$

Therefore, $\tilde{\tau} \circ \tilde{\tau} \nsubseteq \tilde{\tau}$

Therefore, $\tilde{\tau}$ is not type-2 fuzzy transitive relation.

Hence, $\tilde{\tau}$ satisfies all the conditions of type-2 fuzzy G-tolerance relations.

Consequently, $\tilde{\tau}$ is a type-2 fuzzy $G$-tolerance relation. 


\section{Type-2 FUZZY G-TOLERANCE RELATION TO TYPE-2 FUZZY EQUIVALENCE RELATION}

Type-2 fuzzy tolerance relation can be converted to type-2 fuzzy G-equivalence relation by composition. Let us observe this example below:

Example 2. Consider the earlier Example 1

$$
\tilde{\tau}=\left(\begin{array}{ccc}
\frac{1}{1}+\frac{0.9}{0.9} & \frac{0.1}{0.1}+\frac{0.2}{0.2} & \frac{0.4}{0.4}+\frac{0.5}{0.5} \\
\frac{0.1}{0.1}+\frac{0.2}{0.2} & \frac{1}{1}+\frac{0.9}{0.9} & \frac{0.6}{0.7}+\frac{0.6}{0.6} \\
\frac{0.4}{0.4}+\frac{0.5}{0.5} & \frac{0.6}{0.7}+\frac{0.6}{0.6} & \frac{0.7}{0.7}+\frac{0.8}{0.8}
\end{array}\right)
$$
Now,

We conclude that $\tilde{\tau}$ is a type-2 fuzzy G-tolerance relation from Example 1.

$$
\tilde{\tau} \circ \tilde{\tau}=\left(\begin{array}{ccc}
\frac{1}{1}+\frac{0.9}{0.9} & \frac{0.1}{0.1}+\frac{0.2}{0.2} & \frac{0.4}{0.4}+\frac{0.5}{0.5} \\
\frac{0.1}{0.1}+\frac{0.2}{0.2} & \frac{1}{1}+\frac{0.9}{0.9} & \frac{0.6}{0.7}+\frac{0.6}{0.6} \\
\frac{0.4}{0.4}+\frac{0.5}{0.5} & \frac{0.6}{0.7}+\frac{0.6}{0.6} & \frac{0.7}{0.7}+\frac{0.8}{0.8}
\end{array}\right) \circ\left(\begin{array}{ccc}
\frac{1}{1}+\frac{0.9}{0.9} & \frac{0.1}{0.1}+\frac{0.2}{0.2} & \frac{0.4}{0.4}+\frac{0.5}{0.5} \\
\frac{0.1}{0.1}+\frac{0.2}{0.2} & \frac{1}{1}+\frac{0.9}{0.9} & \frac{0.6}{0.7}+\frac{0.6}{0.6} \\
\frac{0.4}{0.4}+\frac{0.5}{0.5} & \frac{0.6}{0.7}+\frac{0.6}{0.6} & \frac{0.7}{0.7}+\frac{0.8}{0.8}
\end{array}\right)
$$

Let us now form a new matrix $\tilde{\tilde{\tau}}$ with the new elements found from the $\tilde{\tau} \circ \tilde{\tau}$. Suppose,

$$
\tilde{\tau}=\left(\begin{array}{ccc}
\frac{0.2}{1}+\frac{0.2}{0.9} & \frac{0.2}{0.4}+\frac{0.2}{0.5} & \frac{0.2}{0.4}+\frac{0.2}{0.5} \\
\frac{0.2}{0.4}+\frac{0.2}{0.5} & \frac{0.2}{1}+\frac{0.2}{0.9} & \frac{0.2}{0.7}+\frac{0.2}{0.6} \\
\frac{0.2}{0.4}+\frac{0.2}{0.5} & \frac{0.2}{0.7}+\frac{0.2}{0.6} & \frac{0.5}{0.7}+\frac{0.5}{0.8}
\end{array}\right) .
$$

We have noticed from the above matrix $\tilde{\tilde{\tau}}$ that it satisfies all the conditions of type-2 fuzzy G-equivalence relation and symmetric relation. We now check whether $\tilde{\tilde{\tau}}$ satisfies the type-2 fuzzy transitive relation.

$$
\begin{aligned}
& \tilde{\tilde{\tau}} \circ \tilde{\tilde{\tau}}=\left(\begin{array}{ccc}
\frac{0.2}{1}+\frac{0.2}{0.9} & \frac{0.2}{0.4}+\frac{0.2}{0.5} & \frac{0.2}{0.4}+\frac{0.2}{0.5} \\
\frac{0.2}{0.4}+\frac{0.2}{0.5} & \frac{0.2}{1}+\frac{0.2}{0.9} & \frac{0.2}{0.7}+\frac{0.2}{0.6} \\
\frac{0.2}{0.4}+\frac{0.2}{0.5} & \frac{0.2}{0.7}+\frac{0.2}{0.6} & \frac{0.5}{0.7}+\frac{0.5}{0.8}
\end{array}\right) \circ\left(\begin{array}{ccc}
\frac{0.2}{1}+\frac{0.2}{0.9} & \frac{0.2}{0.4}+\frac{0.2}{0.5} & \frac{0.2}{0.4}+\frac{0.2}{0.5} \\
\frac{0.2}{0.4}+\frac{0.2}{0.5} & \frac{0.2}{1}+\frac{0.2}{0.9} & \frac{0.2}{0.7}+\frac{0.2}{0.6} \\
\frac{0.2}{0.4}+\frac{0.2}{0.5} & \frac{0.2}{0.7}+\frac{0.2}{0.6} & \frac{0.5}{0.7}+\frac{0.5}{0.8}
\end{array}\right) \\
& \tilde{\tilde{\tau}} \circ \tilde{\tilde{\tau}}=\left(\begin{array}{ccc}
\frac{0.2}{1}+\frac{0.2}{0.9} & \frac{0.2}{0.4}+\frac{0.2}{0.5} & \frac{0.2}{0.4}+\frac{0.2}{0.5} \\
\frac{0.2}{0.4}+\frac{0.2}{0.5} & \frac{0.2}{1}+\frac{0.2}{0.9} & \frac{0.2}{0.7}+\frac{0.2}{0.6} \\
\frac{0.2}{0.4}+\frac{0.2}{0.5} & \frac{0.2}{0.7}+\frac{0.2}{0.6} & \frac{0.2}{0.7}+\frac{0.2}{0.8}
\end{array}\right) \sqsubseteq\left(\begin{array}{ccc}
\frac{0.2}{1}+\frac{0.2}{0.9} & \frac{0.2}{0.4}+\frac{0.2}{0.5} & \frac{0.2}{0.4}+\frac{0.2}{0.5} \\
\frac{0.2}{0.4}+\frac{0.2}{0.5} & \frac{0.2}{1}+\frac{0.2}{0.9} & \frac{0.2}{0.7}+\frac{0.2}{0.6} \\
\frac{0.2}{0.4}+\frac{0.2}{0.5} & \frac{0.2}{0.7}+\frac{0.2}{0.6} & \frac{0.5}{0.7}+\frac{0.5}{0.8}
\end{array}\right) .
\end{aligned}
$$

Consequently, $\tilde{\tilde{\tau}} \circ \tilde{\tilde{\tau}} \sqsubseteq \tilde{\tilde{\tau}}$.

Therefore, $\underset{\tilde{\tau}}{\tilde{\tau}}$ satisfies the transitive property.

Therefore, $\tilde{\tilde{\tau}}$ is a type-2 fuzzy G-equivalence relation.

Definition 8. Let $N$ be an ordinary set and $\tilde{\tau}$ be a type-2 fuzzy G-tolerance relation on $N$. Then a type-2 fuzzy subset $\tilde{Q}$ of $N$ is called a type-2 fuzzy G pre-class iff $\tilde{Q} \times \tilde{Q} \sqsubseteq \tilde{\tau}$ i.e. $\tilde{Q}(x) \wedge \tilde{Q}(y) \sqsubseteq \tilde{\tau}(x, y)$ for all $x, y \in N$.

Example 3. Let $N=\{x, y, z\}$. Then, $\tilde{\tau}$ is a type-2 fuzzy G-tolerance relation on $M$, defined by the following matrix: 


$$
\tilde{\tau}=\left(\begin{array}{ccc}
\frac{1}{1}+\frac{0.9}{0.9} & \frac{0.1}{0.1}+\frac{0.2}{0.2} & \frac{0.4}{0.4}+\frac{0.5}{0.5} \\
\frac{0.1}{0.1}+\frac{0.2}{0.2} & \frac{1}{1}+\frac{0.9}{0.9} & \frac{0.6}{0.7}+\frac{0.6}{0.6} \\
\frac{0.4}{0.4}+\frac{0.5}{0.5} & \frac{0.6}{0.7}+\frac{0.6}{0.6} & \frac{0.7}{0.7}+\frac{0.8}{0.8}
\end{array}\right) .
$$

Let $\tilde{Q}$ be a type-2 fuzzy subset of $N$ defined by $\tilde{Q}(x)=\frac{0.7}{0.7}+\frac{0.8}{0.8}, \tilde{Q}(y)=\frac{0.1}{0.1}+\frac{0.2}{0.2}, \tilde{Q}(z)=\frac{0.3}{0.3}+\frac{0.4}{0.4}$. Then,

$$
\tilde{Q} \times \tilde{Q}=\left(\begin{array}{ccc}
\frac{0.7}{0.7}+\frac{0.8}{0.8} & \frac{0.1}{0.1}+\frac{0.2}{0.2} & \frac{0.3}{0.3}+\frac{0.4}{0.4} \\
\frac{0.1}{0.1}+\frac{0.2}{0.2} & \frac{0.1}{0.1}+\frac{0.2}{0.2} & \frac{0.1}{0.1}+\frac{0.2}{0.2} \\
\frac{0.4}{0.4}+\frac{0.5}{0.5} & \frac{0.1}{0.1}+\frac{0.2}{0.2} & \frac{0.3}{0.3}+\frac{0.4}{0.4}
\end{array}\right)
$$

Clearly, $\tilde{Q} \times \tilde{Q} \sqsubseteq \tilde{\tau}$, and hence is a type-2 fuzzy $G$ pre-class.

There can be another $\tilde{R}$ of $N$ larger than $\tilde{Q}$ such that $\tilde{R}(x) \tilde{\wedge} \tilde{R}(y) \sqsubseteq \tilde{\tau}(x, y)$ e.g. $\tilde{R}(x)=\frac{0.7}{0.7}+\frac{0.8}{0.8}$, $\tilde{R}(y)=\frac{0.1}{0.1}+\frac{0.2}{0.2}, \quad \tilde{R}(z)=\frac{0.5}{0.5}+\frac{0.6}{0.6}$.

Definition 9. Let $\tilde{\tau}$ be a type-2 fuzzy tolerance relation on $N$. A type-2 fuzzy subset $\tilde{C}$ of $N$ is called a type-2 fuzzy $G$-tolerance class if $\tilde{C}$ is a type-2 fuzzy $G$ pre-class and there exists no type-2 fuzzy $G$ pre-class $\tilde{D}$ of $N$ s.t. $\tilde{C} \sqsubset \tilde{D}$.

Example 4. From the Example 3, $\tilde{R}$ is a type-2 fuzzy G-tolerance class while $\tilde{Q}$ is not.

Definition 10. A type-2 fuzzy G-tolerance space is a pair $(M, \tau)$, where $M$ is an ordinary set and $\tau$ is a type-2 fuzzy tolerance relation defined on $M$.

\section{Type-2 Fuzzy Tolerance Relations in Algebraic Structures}

We shall investigate how type-2 fuzzy tolerance relations can be applied in abstract algebra.

Let an algebraic structure $\mathfrak{U}=(A, \mathfrak{F})$ be given, where $A$ is the set of elements of this structure and $\mathfrak{F}$ is the set of operations on this set. If a type- 2 fuzzy tolerance $\tau$ on $A$ is given, we say that $\tau$, is compatible with $\mathfrak{U}$ or $\mathfrak{U}$ is a $\tau$ tolerance algebraic structure if and only if the following holds: Let $f \in \mathfrak{F}$ and let $f$ be an n-ary operation. If we have $2 n$ elements $x_{1}, x_{2}, \ldots, x_{n} ; y_{1}, y_{2}, \ldots, y_{n}$ of $A$ such that $\left(x_{i}, y_{i}\right) \in \tau$ for $i=1,2, \ldots . ., n$, then also $\left(f\left(x_{1}, x_{2}, \ldots, x_{n}\right), f\left(y_{1}, y_{2}, \ldots, y_{n}\right)\right) \in \tau$.

Theorem 5.1. Let $\mathfrak{U}=(A, \mathfrak{F})$ be an algebra and $\tau_{1}, \tau_{2}$ be two type-2 fuzzy tolerance relation on $M$ which are compatible with $U$. Then the relation $\tau_{1} \cap \tau_{2}$ is a type-2 fuzzy tolerance relation compatible with $U$.

Proof. Let $f \in \mathfrak{F}$ be an $n$-ary operation on $U$ and let $x_{1}, x_{2}, \ldots, x_{n} ; y_{1}, y_{2}, \ldots, y_{n}$ be elements of $M$ such that $\left(x_{i}, y_{i}\right) \in \tau_{1} \cap \tau_{2}$ for $i=1,2, \ldots, n$. Then $\left(x_{i}, y_{i}\right) \in \tau_{1}$ and therefore $\left(f\left(x_{1}, x_{2}, \ldots, x_{n}\right), f\left(y_{1}, y_{2}, \ldots, y_{n}\right)\right) \in$ $\tau_{1}$. Similarly, $\left(f\left(x_{1}, x_{2}, \ldots, x_{n}\right), f\left(y_{1}, y_{2}\right.\right.$, $\left.\left.\ldots, y_{n}\right)\right) \in \tau_{2}$. Therefore, $\left(f\left(x_{1}, x_{2}, \ldots, x_{n}\right), f\left(y_{1}, y_{2}, \ldots, y_{n}\right)\right) \in \tau_{1} \cap \tau_{2}$. As $f$ was chosen arbitrarily, this holds for any $f \in \mathfrak{F}$ and $\tau_{1} \cap \tau_{2}$ is a compatible tolerance relation on $U$.

Theorem 5.2. Let $G$ be a group, and let a type-2 fuzzy tolerance $\tau$ be given on its set of elements. If $G$ is a type-2 fuzzy $\tau$ tolerance semigroup with respect to multiplication, it is also a type-2 fuzzy $\tau$ tolerance group.

Proof. Suppose that $G$ is a type-2 fuzzy $\tau$-tolerance semi-group then $\left(x_{1}, y_{1}\right) \in \tau,\left(x_{2}, y_{2}\right) \in \tau$ implies that $\left(x_{1} x_{2}, y_{1} y_{2}\right) \in \tau$ for arbitrary elements $x_{1}, x_{2}, y_{1}, y_{2}$ of G. It is necessary and sufficient to prove that $(x, y) \in \tau$ implies that $\left(x^{-1}, y^{-1}\right) \in \tau$ for $G$ to be a type-2 fuzzy $\tau$-tolerance group for arbitrary elements $x, y$ of $G$. Let us consider two elements $x, y$ of $\mathrm{G}$ and let $(x, y) \in \tau$. Suppose the identity element of the group will be denoted by $e$. Since $\tau$ is a type- 2 fuzzy reflexive, $\left(x^{-1}, x^{-1}\right) \in \tau$. As $(x, y) \in \tau,\left(x^{-1}, x^{-1}\right) \in \tau$, we obtain $\left(x x^{-1}, y x^{-1}\right) \in \tau$, therefore $\left(e, y x^{-1}\right) \in \tau$. Similarly, $\left(y^{-1}, y^{-1}\right) \in$ $\tau$ and $\left(e, y x^{-1}\right) \in \tau$. Therefore, $\left(y^{-1} e, y^{-1} y x^{-1}\right) \in \tau$ which means $\left(y^{-1}, x^{-1}\right) \in \tau$. As $\tau$ is symmetric, also $\left(x^{-1}, y^{-1}\right) \in \tau$. 
Theorem 5.3. Let $G$ be a type-2 fuzzy $\tau$ tolerance group, $e$ its identity element. The set $H$ of the elements $x \in G$ such that $(e, x) \in \tau$ is a normal subgroup of the group $G$.

Proof. Suppose that $x \in H, y \in H$ then according to the statement of the theorem $(e, x) \in \tau,(e, y) \in \tau$. Since, $(e, x) \in \tau,(e, y) \in \tau$, we obtain $(e, x y) \in \tau$. The type-2 fuzzy reflexive relation of $\tau$ implies that $\left(x^{-1}, x^{-1}\right) \in \tau$.We have $(e, x) \in \tau$ and $\left(x^{-1}, x^{-1}\right) \in \tau$ then $\left(x^{-1}, e\right) \in \tau$ or $\left(e, x^{-1}\right) \in \tau$ and consequently $x^{-1} \in H$. Therefore, $\mathrm{H}$ is a subgroup of the group $\mathrm{G}$. Let $z \in G$ and $(z, z) \in \tau$. Since $(e, x) \in \tau$ and $(z, z) \in \tau$ imply $(z, x z) \in \tau$. Again, $\left(z^{-1}, z^{-1}\right) \in \tau$ and $(z, x z) \in \tau$ implies that $\left(e, z^{-1} x z\right) \in \tau$. Hence, $z^{-1} x z \in H$ for any $x \in H$ and $z \in G$. Therefore, $H$ is a normal subgroup of the group $G$.

Theorem 5.4. Let $G$ be a type-2 fuzzy $\tau$ tolerance group, $e$ its identity element. The set $H$ of the elements $x \in G$ such that $(e, x) \in \tau$ is isomorphic to the group $G$.

Proof. According to theorem 3, the set $H$ of all elements $x \in G$ s.t. $(e, x) \in \tau$ is a normal subgroup of the group $G$. Let $x \in H, y \in H$ then according to the statement of the theorem $(e, x) \in \tau$ and $(e, y) \in \tau$. Let $z \in G$ and consider the class $z H$ in the group $G$. Let $x^{\prime} \in z H, y^{\prime} \in z H$. This shows that $x^{\prime}=z x, y^{\prime}=z y$, where $x \in H, y \in H$. Since $x, y \in H$ so $(x, y) \in \tau$. As $z \in G$ and $(z, z) \in \tau$ because $\tau$ is type-2 fuzzy reflexive. Again, $(z, z) \in \tau$ and this together with $(x, y) \in \tau$ implies that $(z x, z y) \in \tau$, thus $\left(x^{\prime}, y^{\prime}\right) \in \tau$. Let us have two elements $z_{1}, z_{2}$ of $G$ such that $z_{1} H \neq z_{2} H$. Let $x_{1} \in z_{1} H, x_{2} \in z_{2} H$. This means that $x_{1}=z_{1} y_{1}, x_{2}=z_{2} y_{2}$ where $y_{1} \in H, y_{2} \in H$. Suppose that $\left(x_{1}, x_{2}\right) \in \tau$ which implies that $\left(z_{1} y_{1}, z_{2} y_{2}\right) \in \tau$. The relations $\left(z_{1}^{-1}, z_{1}^{-1}\right) \in \tau$ and $\left(z_{1} y_{1}, z_{2} y_{2}\right) \in \tau$ imply $\left(y_{1}, z_{1}^{-1} z_{2} y_{2}\right) \in \tau$. Again, $\left(y_{1}, z_{1}^{-1} z_{2} y_{2}\right) \in \tau$ and $\left(y_{1}^{-1}, y_{1}^{-1}\right) \in \tau$ which implies that $\left(e, z_{1}^{-1} z_{2} y_{2} y_{1}^{-1}\right) \in \tau$ and therefore, $z_{1}^{-1} z_{2} y_{2} y_{1}^{-1} \in H$. As $H$ is a subgroup of the group $G$ and the elements $y_{1}, y_{2} \in H$, $\left(z_{1}^{-1} z_{2} y_{2} y_{1}^{-1}\right) y_{1} y_{2}^{-1}=z_{1}^{-1} z_{2} \in H$. But then $z_{2} \in z_{1} H$, [because $z_{2}=z_{1}\left(z_{1}^{-1} z_{2}\right)$ and $z_{1}^{-1} z_{2} \in H$ ] and therefore $z_{1} H=z_{2} H$ which is a contradiction to the assumption that $z_{1} H \neq z_{2} H$.

Theorem 5.5. Let $S$ be a $\tau$ type-2 fuzzy tolerance semi group. T its right(or left, or two sided) ideal. The set $\tau T$ of the elements $x$ of $S$ such that $\left(x, x^{\prime}\right) \in \tau$ where $x^{\prime} \in T$, is a right (or left, or two sided, respectively) ideal of the semi group $S$.

Proof. Let $x \in \tau T$, let $T$ be left ideal of $S$. There exists $x^{\prime} \in T$ such that $\left(x, x^{\prime}\right) \in \tau$. Now, let $y \in S$. As the relation $\tau$ is reflexive, we have $(y, y) \in \tau$. Then $\left(x, x^{\prime}\right) \in \tau,(y, y) \in \tau$ which implies that $\left(x y, x^{\prime} y\right) \in \tau$. But $x^{\prime} y \in T$ because $x^{\prime} \in T$ and $T$ is left ideal. Therefore, $x y \in \tau T$ and $\tau T$ is the left ideal of the semigroup. Similarly, for right and two sided ideal.

\section{Conclusion}

We have introduced the type-2 fuzzy G-tolerance relation and showed that it can result to type-2 fuzzy G-equivalence relations by composition. We have also studied the concept of type-2 fuzzy Gtolerance relations in abstract algebra.

Acknowledgements. The work and research of the second author of this paper is financially supported by National Institute of Technology Silchar, Assam, India.

\section{REFERENCES}

[1] R. Aliev, W. Pedrycz, B. Guirimov, et al., Type-2 fuzzy nueral networks with fuzzy clustering and differential evolution optimization, Inf. Sci. 181 (2011), 1591-1608.

[2] S. Chakravarty and P.K. Dash, A PSO based integrated functional link net and interval type-2 fuzzy logic system for predicting stock market indices, Appl. Soft Comput. 12 (2012), 931-941.

[3] B. Choi and F. Rhee, Interval type-2 fuzzy membership function generation methods for pattern recognition, Inf. Sci. 179 (2009), 2102-2122.

[4] T. Dereli, A. Baykasoglu, K. Altun, et al., Industrial applications of type-2 fuzzy sets and systems: a concise review, Comput. Indust. 62 (2011), 125-137.

[5] Dhiman Dutta and Mausumi Sen, Type-2 fuzzy equivalence relation on a groupoid under balanced and semibalanced maps, J. Inf. Math. Sci. (2017), in press .

[6] D. Dubois and H. Prade, Operations on fuzzy numbers, Int. J. Syst. Sci. 9 (6) (1978), 613-626.

[7] D. Dubois and H. Prade, Operations in a fuzzy-valued Logic, Inf. Control 43 (1979), 224-240. 
[8] D. Dubois and H. Prade, Fuzzy Sets and Systems: Theory and Applications, first ed., Acdemic Press Inc., New York, 1980.

[9] S.S. Gilan, M.H. Sebt and V. Shahhosseini, Computing with words for hierarchical competency based selection of personal in construction companies, Appl. Soft Comput. 12 (2012), 860-871.

[10] B. Q. Hu and C. Y. Wang, On type-2 fuzzy relations and interval-valued type-2 fuzzy sets, Fuzzy Sets Syst. 236 (2014), 1-32.

[11] N.N. Karnik and J.M. Mendel, Applications of type-2 fuzzy logic systems to forecasting of time-series, Inf. Sci. 120 (1999), 89-111.

[12] N. N. Karnik and J. M. Mendel, Operations on type-2 fuzzy sets, Fuzzy Sets Syste. 79 (2001), 327-348.

[13] P. Kundu, S. Kar and M. Maiti, Multi-item solid transportation problem with type-2 fuzzy parameters, Appl. Soft Comput. 31 (2015), 61-80.

[14] J.M. Mendel, Computing with words and its relationship with fuzzistics, Inf. Sci. 177 (2007), 988-1006.

[15] M.Mizumoto and K.Tanaka, Some Properties of fuzzy sets of type-2, Inf. Control 31 (1976), 312-340.

[16] M.Mizumoto and K.Tanaka, Fuzzy sets of type-2 under algebraic product and algebraic sum, Fuzzy Sets Syst. 31 (1981), 277-290.

[17] I. Ozkan and I.B. Turksen, MiniMax e-star cluster validity index for type-2 fuzziness, Inform. Sci. 184 (2012), 64-74.

[18] C. Leal-Ramirez, O. Castillo, P. Melin, et al., Simulation of the bird age-structured population growth based on an interval type-2 fuzzy cellular structure, Inf. Sci. 181 (2011), 519-535.

[19] S.W. Tung, C. Quek, C. Guan, eT2FIS: an evolving type-2 neural fuzzy inference system, Inf. Sci. 220 (2013), 124-148.

[20] D. Wu, W. Tan, A simplified type-2 fuzzy logic controller for real-time control, ISA Trans. 45 (4) (2006), 503-516.

[21] L.A. Zadeh, The Concept of a Linguistic Variable and its Application to Approximate Reasoning-I, Inf. Sci. 8 (1975), 199-249.

${ }^{1}$ Department of Mathematics, National Institute of Technology Silchar, Assam, India

${ }^{2}$ Berkeley Initiative in Soft Computing, University of California, Berkeley CA USA, Department of Computer Science and Engineering, National Institute of Technology Silchar, Silchar, India

*Corresponding AUthor: senmausumi@gmail.com 